\title{
Management of large inflammatory dentigerous cysts adapted to the general condition of the patient: Two case reports
}

\author{
ARINA VINEREANU $^{1 *}$, ANDRADA BRATU $^{2}$, ANDREEA DIDILESCU $^{3 *}$ and ANETA MUNTEANU ${ }^{1 *}$ \\ ${ }^{1}$ Department of Pedodontics, 'Carol Davila' University of Medicine and Pharmacy, Faculty of Dental Medicine, \\ 010221 Bucharest; ${ }^{2}$ Private Practice, 060015 Bucharest; ${ }^{3}$ Department of Embryology, \\ 'Carol Davila' University of Medicine and Pharmacy, 010221 Bucharest, Romania
}

Received March 9, 2021; Accepted April 8, 2021

DOI: $10.3892 /$ etm.2021.10182

\begin{abstract}
Dentigerous cysts may be of developmental or inflammatory origin. The latter occur in unerupted permanent teeth as a result of inflammation from a preceding non-vital primary tooth or from another source spreading to involve the tooth follicle. This report presents two clinical cases of children with dentigerous cysts of inflammatory origin. Case 1 is a healthy boy (7 years 11 months) referred for a large cystic cavity in the right mandibular premolar region. Extraction of 84 and 85 and marsupialization of the cyst were performed under nitrous sedation. A removable appliance with an acrylic piece fitted into the socket was applied on the same occasion. The in-socket piece was progressively reduced as the cystic cavity was shrinking. After a 20-month follow-up, 44 and 45 are sound and correctly erupted and 46 remains unaffected. Case 2 is an autistic girl (10 years 9 months) with bilateral large odontogenic cysts enclosing the crowns of 35 and 45. Extractions of 75, 85 were performed under general anesthesia, leaving large bone defects. Given the limited compliance of the patient under common dental office circumstances, no appliance was used. Thirteen months after extraction, 35 and 45 are sound, fully erupted and no visible mesial drifting of 36 and 46 occurred. In conclusion, conservative treatment of large inflammatory dentigerous cysts in children gives good results with minimal intervention, ensures physiologic development of teeth and proper bone healing. The general condition of the patient can influence treatment choice. Patients must be followed up until eruption of the displaced permanent teeth and bony consolidation of the cyst.
\end{abstract}

Correspondence to: Dr Arina Vinereanu, Department of Pedodontics, 'Carol Davila' University of Medicine and Pharmacy, Faculty of Dental Medicine, 17-21 Calea Plevnei, 010221 Bucharest, Romania

E-mail: avinereanu2002@yahoo.co.uk

*Contributed equally

Key words: inflammatory dentigerous cysts, children, conservative treatment, marsupialization, removable appliance, general condition

\section{Introduction}

Dentigerous cyst is a type of odontogenic cyst and accounts for $20-24 \%$ of all jaw cysts (1). There are two types of dentigerous cysts: one of developmental origin, associated with impacted teeth (2) and the other one, of inflammatory origin, associated with non-vital primary teeth $(3,4)$. Inflammatory dentigerous cysts are only found in the mixed dentition and they generally involve mandibular premolars $(3,5)$.

A dentigerous cyst encloses the crown of an unerupted tooth by expansion of its follicle and is attached to the tooth along the cervical region (6). Dentigerous cysts are often asymptomatic and they are usually detected during routine radiographic examination, X-rays taken for orthodontic reasons or in order to find the reason for delayed eruption $(2,7,8)$. In the radiographic examination, dentigerous cyst lesion shows a well-demarcated unilocular radiolucency with a sclerotic border, surrounding the crown of an unerupted tooth $(3,9)$.

The cyst may cause swelling, tooth displacement, tooth mobility and sensitivity if it reaches a size larger than $2 \mathrm{~cm}$ in diameter (7).

Odontogenic keratocyst, unicystic ameloblastoma, central giant cell granuloma and a large radicular cyst must be considered in the differential diagnosis of a dentigerous cyst. Radiograph alone cannot differentiate the above-mentioned lesions, thus a histopathological examination can help accurate diagnosis $(1,3)$.

Histologically, dentigerous cysts consist of a fibrous wall containing variable amounts of myxoid tissue and odontogenic remnants. The cyst is lined with nonkeratinized stratified squamous epithelium consisting of muco-sebaceous, ciliated and, rarely, sebaceous cells. The epithelial-connective tissue interface is typically flattened, but becomes highly irregular when associated with inflammation (10). Pseudoepitheliomatous hyperplasia with thicker epithelium and acute and chronic inflammatory infiltrate are frequently found (8).

Extraction of non-vital primary teeth and marsupialization represent the best strategy to conserve teeth affected by a dentigerous cyst and to permit their eruption, especially in young patients $(1,3,5)$. When unerupted third molars are involved, coronectomy (intentional partial odontectomy) can be a solution $(11,12)$. There are currently more published reports on odontogenic cysts found in adults than in pediatric patients (4). 
This report presents two clinical cases of children with dentigerous cysts of inflammatory origin. The two cases were managed differently due to the associated medical condition of one of the patients who had autism, which limited patient cooperation under common dental office circumstances.

\section{Case reports}

Case 1. A boy aged 7 years 11 months was referred by a fellow surgeon for a second opinion for the treatment of an odontogenic cyst on the right side of the mandible (Fig. 1). General examination and anamnesis confirmed that the patient was healthy, with no significant medical history. Clinical intraoral examination revealed a right lower first permanent molar (46) with untreated caries, while the second primary molar in the same quadrant (85) was mobile, with a large composite restoration. According to anamnesis, 85 had been treated endodontically a few months before. Radiographic examination showed a large unilocular radiolucent area surrounding the crown of the mandibular second premolar (45). The roots of the non-vital second primary molar exhibited accelerated resorption and appeared to project into the lumen of the cystic cavity.

The computed tomography (CT) examination previously recommended by the referring colleague (Fig. 2A and B) revealed a large cystic cavity $(14 \times 12 \mathrm{~mm})$, enclosing the crown of unerupted 45 and displacing medially the crown of unerupted 44. The second premolar was shifted to the lower edge of the mandible.

A previous panoramic X-ray, taken 14 months before referral (Fig. 3), showed deep untreated caries on both lower primary molars on the right side.

Treatment. As a first step, an alginate impression of the lower arch was taken. Teeth 84 and 85 were removed from the cast and a removable lower appliance was custom-made, with a space maintainer corresponding to the removed primary molars. Extractions were scheduled to be performed under inhalation sedation due to the high level of dental anxiety of the child (initial level 2 of cooperation on Frankl scale).

Extraction of both primary molars on the right side and marsupialization of the cyst were performed under nitrous sedation and local infiltration anesthesia. The removable appliance was adapted on site, with an acrylic piece fitted into the socket (Fig. 4A-D).

Parents were instructed to daily irrigate the operative site with saline after the extraction for the next week. Patient was instructed to wear the appliance at all times and was recalled after 7 days and then every 2 weeks.

Follow-up. The in-socket piece was progressively reduced as the cystic cavity was shrinking. After 2 months, 44 regained its upward position and 45 moved towards correct eruption (Fig. 5A and B).

Orthodontic treatment with removable appliances was then initiated due to crowding. Twenty months after surgery, both premolars on the right side were sound and correctly erupted, while on the left side of the mandible deciduous molars were still in place. The right lower first permanent molar remained unaffected despite its proximity to the cyst (Figs. 6 and 7).
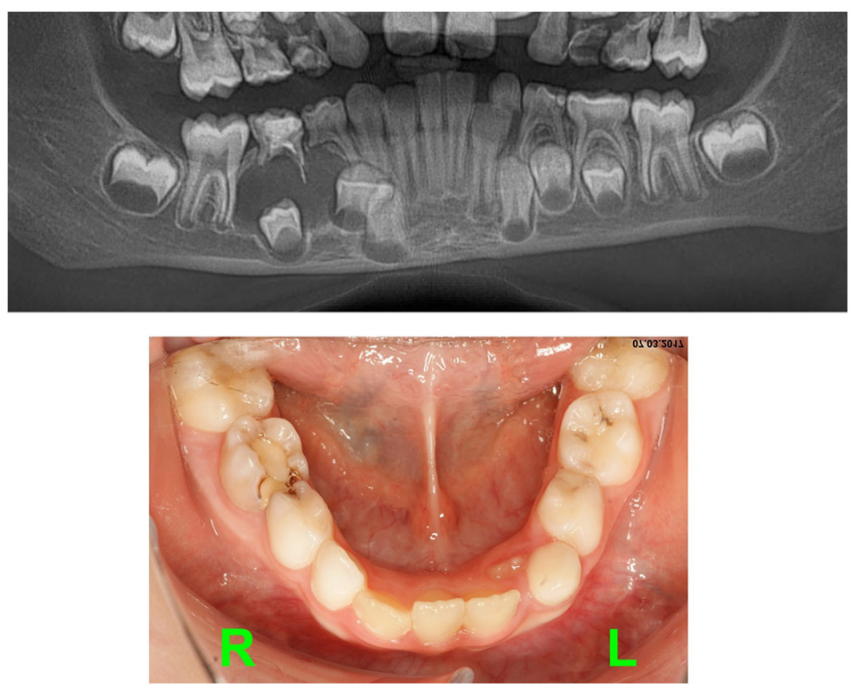

Figure 1. Panoramic X-ray and clinical view at first visit (7/03/2017) of case 1.
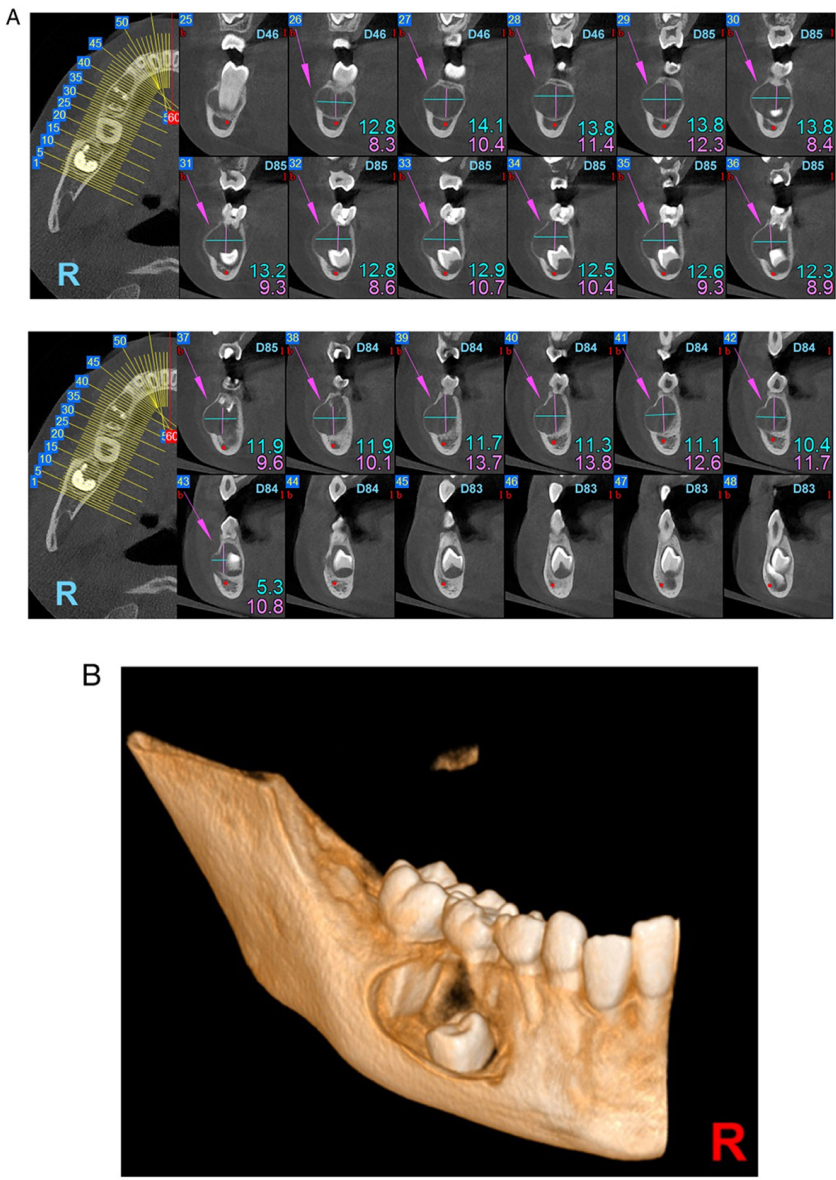

Figure 2. (A and B) Computed tomography (CT) at first visit (7/03/2017) for case 1.

Case 2. In January 2018, bilateral inflammatory follicular cysts were diagnosed during a routine dental check-up in an autistic girl aged 10 years 9 months, treated and followed in our clinic. The vestibular bone plate was slightly deformed. The patient did not exhibit any manifestations that might have suggested pain and there were no clinical signs of inflammation around 


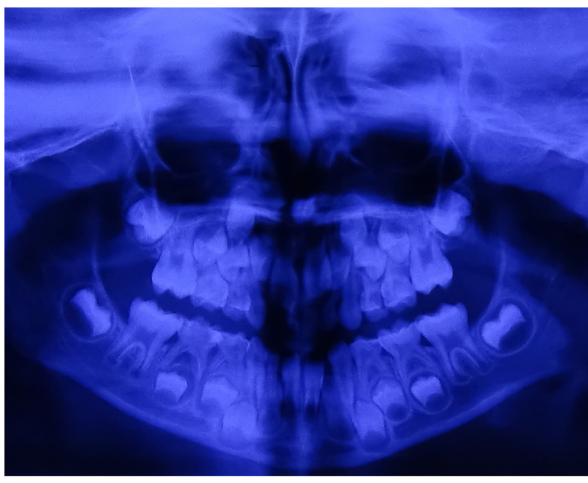

Figure 3. Panoramic X-ray taken 14 months before referral (25/01/2016) for case 1
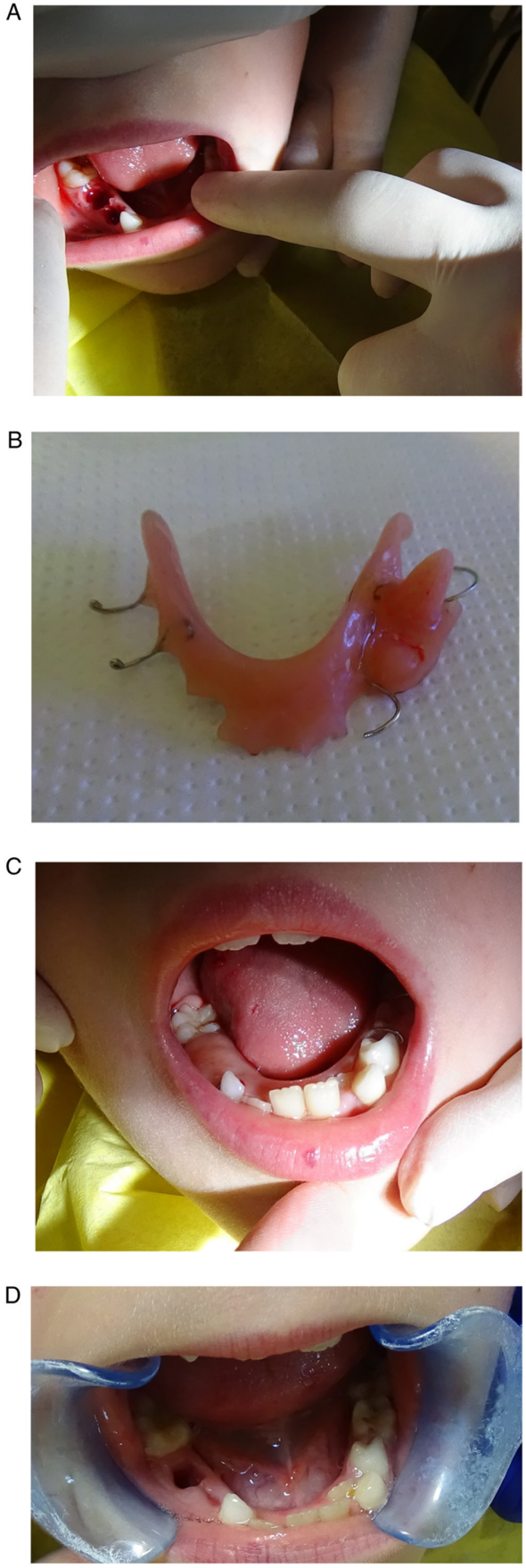

Figure 4. (A) Extraction, (B) appliance, (C) appliance fitting and (D) clinical view after 3 weeks for case 1 .
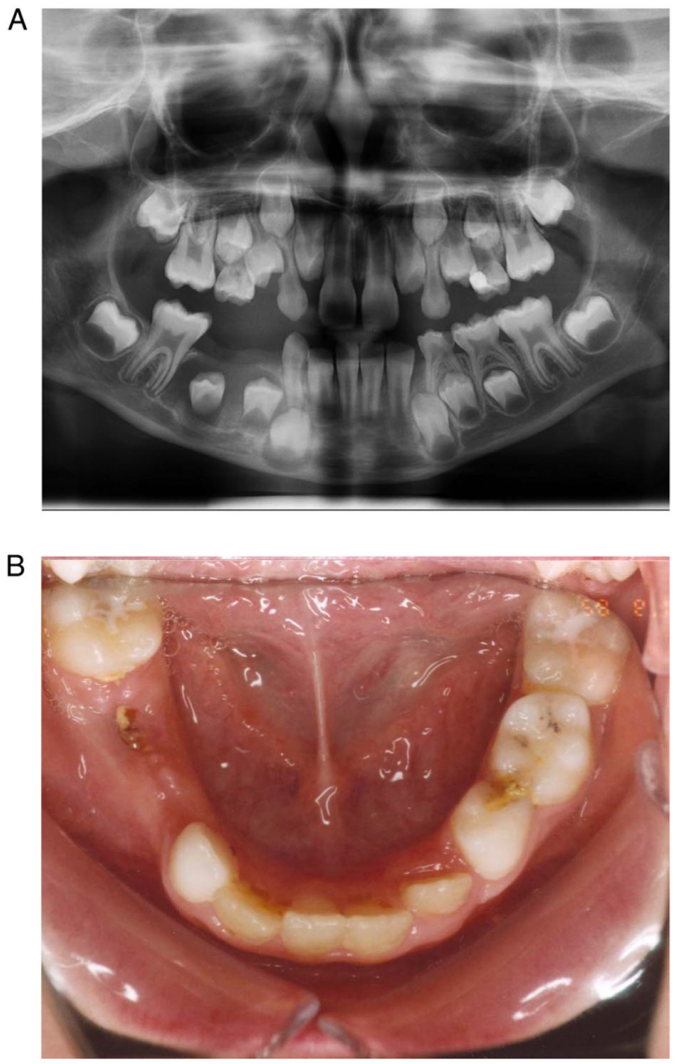

Figure 5. (A) Panoramic X-ray and (B) clinical view 2 months after surgery for case 1 .

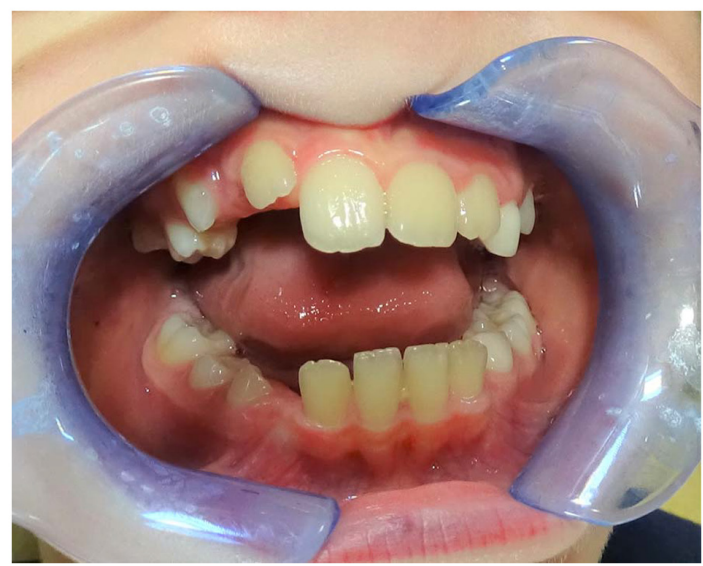

Figure 6. Clinical aspect 14 months after surgery for case 1.

the lesion. Panoramic X-ray showed bilateral large dentigerous cysts enclosing the crowns of the lower second premolars on both sides (Fig. 8B). No other investigations were performed. According to the dental records of the child, endodontic treatment on 75 and 85 had been performed under general anesthesia 33 months before, due to extensive caries (Figs. 8A and 9A and B).

Treatment. Teeth 75 and 85 (together with other 4 primary teeth: 53, 55, 63 and 65) were extracted under general anesthesia (Fig. 10), leaving large bone defects. Given the developmental stage of the lower second premolars, an appliance with an 


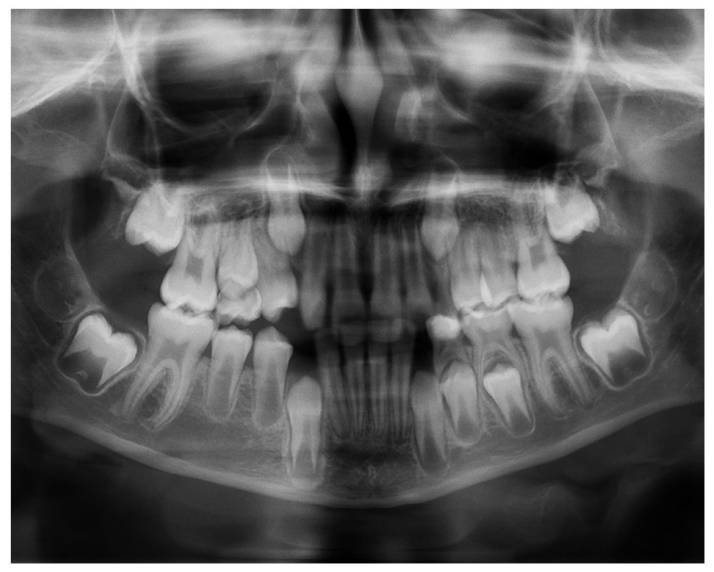

Figure 7. Panoramic X-ray 20 months after surgery for case 1.
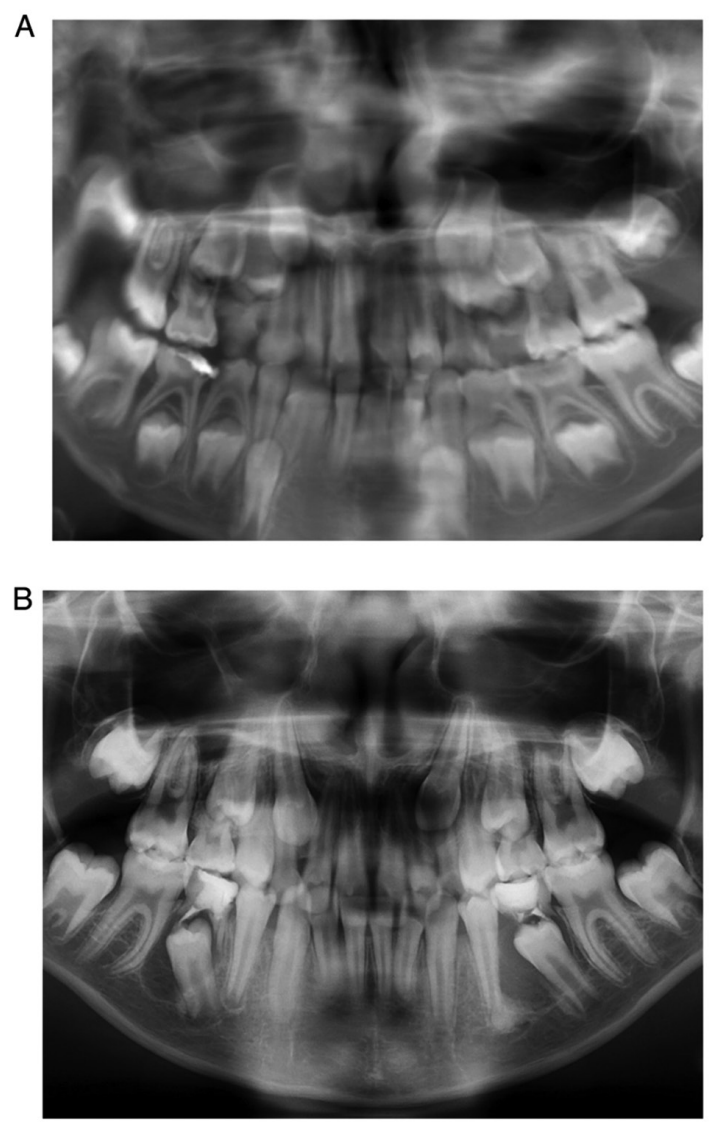

Figure 8. Panoramic X-rays before (A) first and (B) second general anesthesia in case 2.

in-socket piece was not considered necessary. Despite some estimated risk for mesial tilting of the lower first permanent molars, the patients' general pathology ruled out space maintainers as well.

Follow-up. Clinical evolution was good. Thirteen months after extraction, lower second premolars were fully erupted and sound, while 36 and 46 remained in fairly good position (Fig. 11A-C). No further X-rays were taken due to difficulties in obtaining the patient's cooperation, as well as to questionable objective reasons to insist.
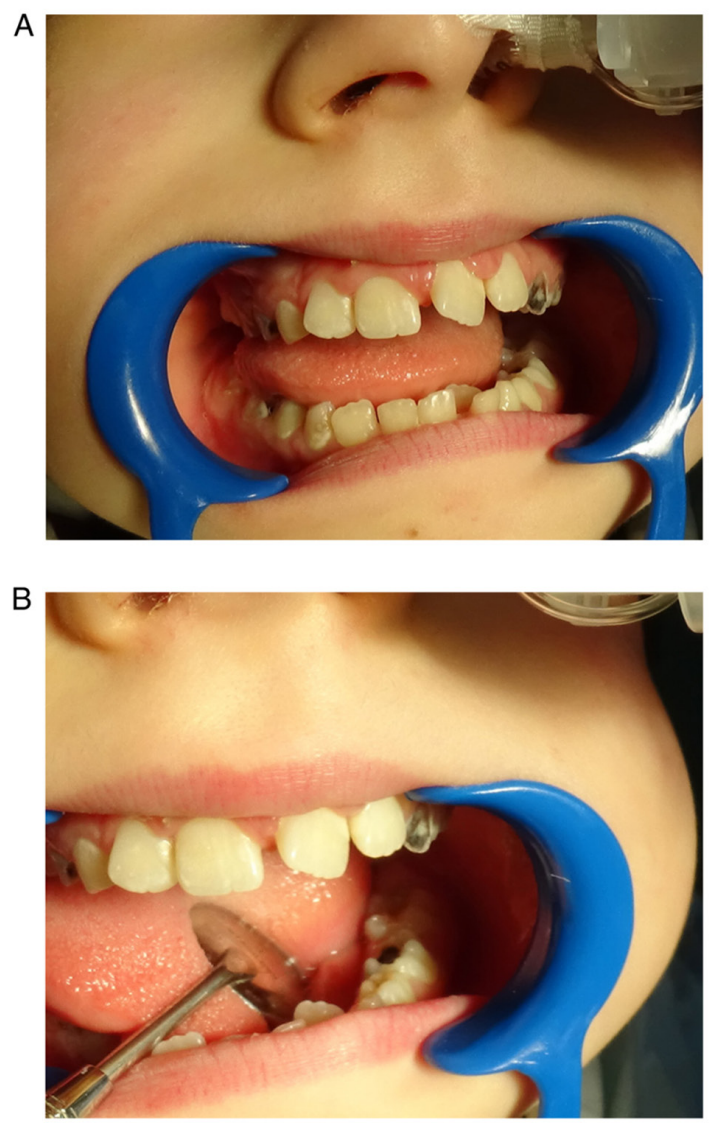

Figure 9. (A and B) Clinical aspects before first general anesthesia (age 7 years 11 months) in case 2 ; multiple, extensive caries were present, requiring comprehensive treatment under general anesthesia.

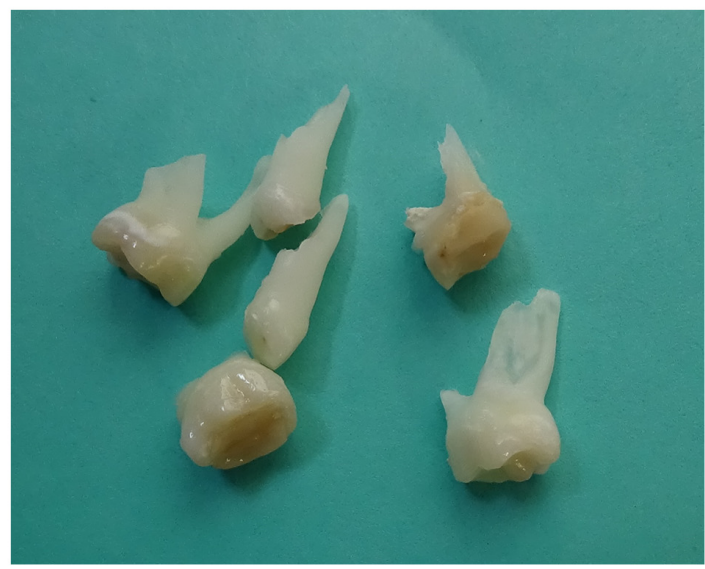

Figure 10. Teeth 55, 53, 63, 75, 85 were extracted under general anesthesia $(26 / 01 / 2018)$ in case 2 .

\section{Discussion}

Inflammatory follicular cysts occur most frequently in the premolar region, after primary molars affected by deep caries become non-vital. Inflammatory follicular cysts occur more frequently in boys than girls and are 10 times more likely to occur in the lower jaw than in the upper (3).

The pathogenesis of dentigerous cysts is still controversial. Benn and Altini (13) proposed three possible mechanisms for 

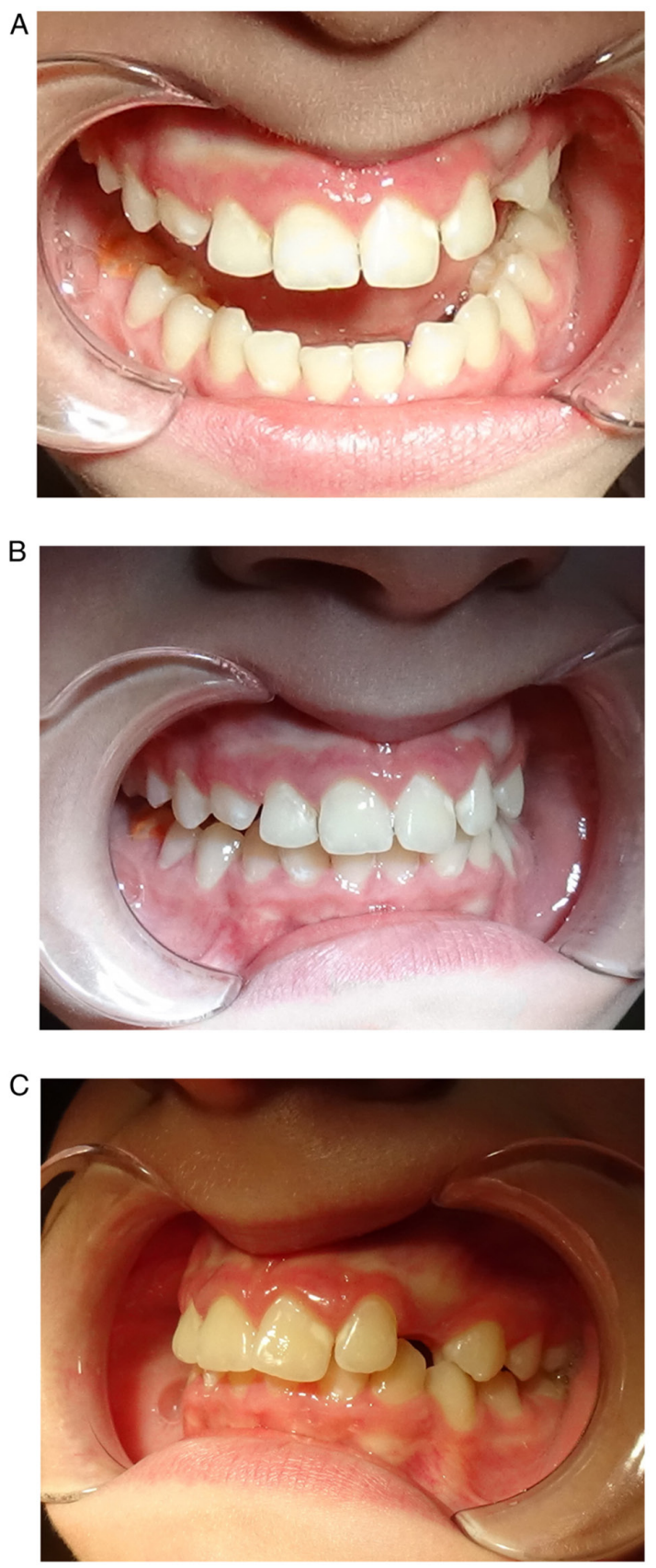

Figure 11. (A-C) Clinical views 13 months after second general anesthesia (needed for the extraction of 75,85 ) in case 2 .

cyst histogenesis. The first hypothesis suggests that the developmental dentigerous cyst might form from a dental follicle, secondarily inflamed from a non-vital tooth as a source of inflammation. The second proposed explanation would be the formation of a radicular cyst at the apex of a non-vital deciduous tooth, followed by eruption of its permanent successor into the radicular cyst, thus resulting in a dentigerous cyst of extrafollicular origin. They also suggested that the follicle of a permanent successor might get secondarily infected from other sources than the non-vital deciduous predecessor, leading to a dentigerous cyst formation. Available evidence in both our cases indicated that infection of the predecessors (second primary molars) could have been the source of inflammation of the dentigerous cysts.
Pulp treatment cannot prevent the development of these cysts as they have been noted to occur both after pulp treatment and in the absence of it (3). In our cases, inflammatory dentigerous cysts occurred in primary molars with endodontic treatment.

Modern imaging [such as cone-beam computed tomography (CBCT)] can provide useful information for the diagnostic and clinical management of dentigerous cysts, especially for large ones, with displacement of adjacent teeth (7). In our first clinical case, the $\mathrm{CT}$ investigation recommended by the referring colleague was very helpful for quick diagnostic and efficient decision-taking. However, careful evaluation of the benefits and need for such investigations compared with the potential risks of exposure to ionizing radiation is extremely important in pediatric patients. The European Academy of Paediatric Dentistry's guidelines for prescribing radiographs in children and adolescents clearly point out that CBCT examination can be justified only when it results in a substantial net gain of information which directly influences the decision-making (14). Besides the age of the patient and the expected diagnostic benefits, the child's capacity to cooperate for complex imaging (needing a longer acquisition time) is also to be taken into account when indicating such investigations, especially when general conditions (e.g., intellectual challenge) are present (14).

Both of our clinical cases provide information on the growth rate of inflammatory dentigerous cysts: In the first case, the cyst reached a diameter of $1.4 \mathrm{~cm}$ in 14 months, while in the second case cysts with diameters $>1 \mathrm{~cm}$ developed over a period of $\sim 3$ years. Shear (6) reported radiological findings showing that dentigerous cysts of $4-5 \mathrm{~cm}$ in diameter may develop in 3-4 years. Early recognition of dentigerous cysts, often as incidental findings, and prompt treatment limit the extent of jaw bone destruction (4). Uneventful healing with spontaneous gradual filling of the residual cavities, without any graft material, can be obtained even in large cysts after marsupialization if the cavity is sufficiently surrounded by bony walls (15).

Although rarely, untreated dentigerous cysts may sometimes develop into an odontogenic tumor or a malignancy such as squamous cell carcinoma (1). Therefore, early diagnosis and treatment of a dentigerous cyst lesion is important for the prevention of development into more destructive lesions.

It is generally accepted that extraction of a non-vital primary tooth and marsupialization will allow rapid healing of the lesion and eruption of the permanent tooth, provided that these procedures are performed at the normal time of eruption (3). However, opinions that the treatment plan for dentigerous cysts should consider extraction of the involved permanent tooth are not completely ruled out. In this respect, removal of the associated permanent tooth along with cyst enucleation may be necessary if it shows arrested development, is severely displaced or if the lesion is extensive $(3,5)$.

By extracting the infected primary teeth, marsupialization of the cyst and ensuring continuous drainage, it is possible to achieve spontaneous eruption of the involved permanent teeth even if they are badly dislocated. Healing and ossification of the bony defect can take place simultaneously with the eruption of the permanent teeth. The reparatory process is completed in one to two years, depending on the dimensions of the bone defect (15). 
In our first case, drainage was maintained with a custom-made removable appliance (modified Howley plate), which also played the role of a space maintainer, prevented food impaction and allowed access for regular cleansing. The same method has been successfully used in previous reports (16).

No evidence of recurrence was noted on follow-up radiography after marsupialization. These results are consistent with previous studies, which have shown that recurrence is rare after total lesion removal or when the involved tooth erupts after marsupialization (1,15-17).

In conclusion, conservative treatment of large inflammatory dentigerous cysts in young patients provides good results with minimal invasion, ensures preservation and physiologic development of teeth and proper bone healing. Modern imaging can substantially help diagnostic and clinical decisions, but indications for high radiation investigations must be carefully evaluated. Patients must be followed-up until full eruption of the displaced permanent teeth and bony consolidation of the cyst.

\section{Acknowledgements}

Not applicable.

\section{Funding}

This study was supported by Erasmus+ Project 2019-1-RO01KA202-063820-OSCAR (Oscar Special Care Academic Resources).

\section{Availability of data and materials}

All patient data mentioned in the article are available from the corresponding author on reasonable request.

\section{Authors' contributions}

$\mathrm{AV}$ and $\mathrm{AB}$ provided clinical management of the patients. AV, $\mathrm{AM}$ and $\mathrm{AD}$ contributed in all the stages of the article preparation, from literature research to revising the manuscript for important intellectual content. All authors read and approved the final manuscript.

\section{Ethics approval and consent to participate}

Not applicable.

\section{Patient consent for publication}

Written consent for the publication of the images was obtained from the patients' parents before paper submission.

\section{Competing interests}

The authors declare that they have no competing interests.

\section{Authors' information}

Arina Vinereanu: https://orcid.org/0000-0001-9745-1315.

\section{References}

1. Kirtaniya BC, Sachdev V, Singla A and Sharma AK: Marsupialization: A conservative approach for treating dentigerous cyst in children in the mixed dentition. J Indian Soc Pedod Prev Dent 28: 203-208, 2010.

2. Sindi AM: Bilateral mandibular dentigerous cysts presenting as an incidental finding: A case report. Am J Case Rep 20: 1148-1151, 2019.

3. Koželj V and Sotošek B: Inflammatory dentigerous cysts of children treated by tooth extraction and decompression-report of four cases. Br Dent J 187: 587-590, 1999.

4. Rajendra Santosh AB: Odontogenic cysts. Dent Clin North Am 64: 105-119, 2020.

5. Shetty RM and Dixit U: Dentigerous cyst of inflammatory origin. Int Clin J Pediatr Dent 3: 195-198, 2010.

6. Shear M: Cysts of the oral regions. 3rd edition. Blackwell Munksgaard Publishers, Oxford Wright, pp75-89, 1992.

7. Bodner L, Woldenberg Y and Bar-Ziv J: Radiographic features of large cysts lesion of jaws in children. Pediatr Radiol 33: 3-6, 2003.

8. Huang G, Moore L, Logan RM and Gue S: Histological analysis of 41 dentigerous cysts in a paediatric population. J Oral Pathol Med 48: 74-78, 2019.

9. Ziccardi VB, Eggleston TI and Schnider RE: Using fenestration technique to treat a large dentigerous cyst. J Am Dent Assoc 128: 201-205, 1997.

10. Tüzüm MS: Marsupialization of a cyst lesion to allow tooth eruption: A case report. Quintessence Int 28: 283-284, 1997.

11. Henien M, Sproat C, Kwok J, Beneng K and Patel V: Coronectomy and dentigerous cysts: A review of 68 patients. Oral Surg Oral Med Oral Pathol Oral Radiol 123: 670-674, 2017.

12. Patel V, Sproat C, Samani M, Kwok J and McGurk M: Unerupted teeth associated with dentigerous cysts and treated with coronectomy: Mini case series. Br J Oral Maxillofac Surg 51: 644-649, 2013.

13. Benn A and Altini M: Dentigerous cysts of inflammatory origin. A clinicopathologic study. Oral Surg Oral Med Oral Pathol Oral Radiol Endod 81: 203-209, 1996.

14. Kühnisch J, Anttonen V, Duggal MS, Spyridonos ML, Rajasekharan S, Sobczak M, Stratigaki E, Van Acker JWG, Aps JKM, Horner K and Tsiklakis K: Best clinical practice guidance for prescribing dental radiographs in children and adolescents: An EAPD policy document. Eur Arch Paediatr Dent 21: 375-386, 2020.

15. Chacko R, Kumar S, Paul A and Arvind: Spontaneous bone regeneration after enucleation of large jaw cysts: A digital radiographic analysis of 44 consecutive cases. J Clin Diagn Res 9: ZC84-ZC89, 2015.

16. Nohra J, Kassir AR, Akel H and Dagher M: Treatment of dentigerous cysts with a modified Hawley plate in children: Report of two cases with radiographic results. Br J Oral Maxillofac Surg 58: 102-104, 2020.

17. Koca H, Esin A and Aycan K: Outcome of dentigerous cysts treated with marsupialization. J Clin Pediatr Dent 34: 165-168, 2009. 